\title{
Conscious and unconscious thought in risky choice: testing the capacity principle and the appropriate weighting principle of unconscious thought theory
}

\section{Nathaniel J. S. Ashby *, Andreas Glöckner and Stephan Dickert}

Max Planck Institute for Research on Collective Goods, Bonn, Germany

\section{Edited by:}

Michael Dougherty, University of Maryland at College Park, USA

\section{Reviewed by:}

Adam Goodie, University of Georgia, USA

Timothy Pleskac, Michigan State University, USA

Marius Usher, Tel-Aviv University and Birkbeck College, Israel

\section{${ }^{*}$ Correspondence:}

Nathaniel J. S. Ashby, Max Planck Institute for Research on Collective Goods, Kurt-Schumacher-Str. 10,

D-53113 Bonn, Germany.

e-mail:ashby@coll.mpg.de

\begin{abstract}
Daily we make decisions ranging from the mundane to the seemingly pivotal that shape our lives. Assuming rationality, all relevant information about one's options should be thoroughly examined in order to make the best choice. However, some findings suggest that under specific circumstances thinking too much has disadvantageous effects on decision quality and that it might be best to let the unconscious do the busy work. In three studies we test the capacity assumption and the appropriate weighting principle of Unconscious Thought Theory using a classic risky choice paradigm and including a "deliberation with information" condition. Although we replicate an advantage for unconscious thought (UT) over "deliberation without information," we find that "deliberation with information" equals or outperforms UT in risky choices. These results speak against the generality of the assumption that UT has a higher capacity for information integration and show that this capacity assumption does not hold in all domains. Furthermore, we show that "deliberate thought with information" leads to more differentiated knowledge compared to UT which speaks against the generality of the appropriate weighting assumption.
\end{abstract}

Keywords: unconscious thought, judgment and decision making, rational choice, risky choice

\section{INTRODUCTION}

In a series of experiments investigating hypothetical automobile purchases to diagnosing psychological disorders, Dijksterhuis and colleagues have shown that individuals who are distracted from conscious deliberation fair as well as, and in many cases better than, those who consciously explore the options before making a choice (e.g., de Vries et al., 2010; Dijksterhuis et al., 2010). They propose that in complex decisions people should not interfere with automatic information processes and should instead rely on unconscious processing. The unconscious thought effect (UTE) is both from a practical and theoretical perspective an interesting phenomenon and has inspired a great deal of research and controversial debate. Dijksterhuis and Nordgren (2006) have suggested the Unconscious Thought Theory (UTT) as a general model that can account for the effect. UTT consists of six rather general assumptions (principles) concerning information processing in judgment and decision making. Two of the core assumptions are the capacity principle (i.e., unconscious thought (UT) has the capacity to integrate more information than conscious thought) and the appropriate weighting principle (i.e., UT assigns more appropriate weights to attributes or cues than conscious thought). The generality of these assumptions are tested in this paper and the principles are explained in greater detail below.

In line with the theory, several experiments show that unconscious information processing can lead to the detection and choice of superior options (e.g., Dijksterhuis et al., 2006; Bos et al., 2008; Smith et al., 2008; Ham et al., 2009; Strick et al., 2010; Usher et al., 2011; for a review and meta-analysis see Strick et al., unpublished) although other studies were not successful in replicating this effect (for details see below). Unconscious thought is proposed to be successful by avoiding pitfalls of conscious deliberation (Dijksterhuis, 2004) such as memory retrieval errors (Wilson and Schooler, 1991; Wilson et al., 1993; Reyna and Brainerd, 1995), processing capacity issues (Miller, 1956), and reliance on (and biases caused by) heuristics (Tversky and Kahneman, 1974; Tordesillas and Chaiken, 1999). Nevertheless, UTT remains partially unspecific concerning the boundaries of conscious cognitive capacity, and particularly the cognitive mechanisms behind unconscious processing are not clearly specified. ${ }^{1}$

The classic UTT experiment consists of 4 choice options with 12 associated attributes which are displayed for a limited period of time before participants are placed in one of three conditions: unconscious deliberation, conscious deliberation, or immediate decision. In the unconscious deliberation condition participants are distracted from thinking about the options further via simple tasks before making a decision. In the conscious deliberation condition participants think about the options without the information being available to them before making a selection between choice options. Finally, those in the immediate decision condition make their selection directly after viewing the attributes.

\footnotetext{
${ }^{1}$ This problem of insufficient process specification is thereby not unique to UTT. It is shared by many dual-process models (see Evans, 2008, for a review). Theorizing concerning UTT and the latter approaches could highly benefit from more directly taking into account formal models of memory (e.g., Dougherty et al., 1999; Thomas et al., 2008) and/or perception (e.g., McClelland and Rumelhart, 1981; Usher and McClelland, 2001; Glöckner and Betsch, 2008) that have been applied to judgment and choice in previous research (see Glöckner and Witteman, 2010, for a critical discussion).
} 
People in the unconscious deliberation condition were shown to make the best selection more frequently than those in the conscious deliberation and immediate decision conditions (e.g., Dijksterhuis et al., 2006; Strick et al., unpublished; Strick et al., 2010; Ham et al., 2009; de Vries et al., 2010) lending support for the UTE. Nevertheless, other studies have also repeatedly failed to replicate the effect (Calvillo and Penaloza, 2009; Rey et al., 2009; Thorsteinson and Withrow, 2009; for a critical review see Acker, 2008). Besides the problem that the effect does not always replicate, several authors have cast doubt on the methodological veracity of experiments employing the classic UTT paradigm (GonzálezVallejo et al., 2008; Waroquier et al., 2009, 2010; González-Vallejo and Phillips, 2010). Waroquier et al. (2009) argue that in several studies (Dijksterhuis et al., 2006; Bos et al., 2008; Strick et al., 2010) the material was suboptimal in that the attributes chosen to make one option better than another were poorly constructed. Although this criticism is valid some recent studies have taken further precautions to avoid this confound to the greatest extent possible and still provided replication of the UTE (Usher et al., 2011). This clearly indicates that the UTE cannot be merely attributed to issues of material construction. Payne et al. (2008) attempted to resolve potential problems with the material more fundamentally by using a different kind of tasks, namely by employing decisions between incentivized gambles. As gambles have a clear best option (i.e., the one with the highest expected value (EV) or expected utility (EU)) gamble selection decisions potentially provided a better test of the UTE by having a clear-cut and (when assuming risk neutral preferences) person-independent outside criterion for the goodness of a decision. ${ }^{2}$ Payne et al. (2008) found in two experiments that participants in the UT condition did not show superior choice behavior when compared to those in a self paced conscious thought condition. It should, however, be noted that in response to the critical findings by Payne et al. (2008), Dijksterhuis et al. (2010) argued that the unconscious does not work with numerical information providing a boundary condition for the UTE. In most paradigms used in the literature (e.g., the price and size of a flat or the speed of a car) and also in most real-world decisions numbers are involved. So that taking this argument seriously would limit the scope of the theory to very few - if any - situations. So we prefer to leave it to empirical testing whether parts of the UTE can also be found for risky choices involving gambles.

While the existence of UTE has been explored in depth there is a lack of research directly investigating the core principles of the underlying theory used to explain it. We set out to test two of the key assumptions in UTT that refer to properties of UT: the capacity principle and the appropriate weighting principle.

The capacity principle in UTT is stated very generally. "According to the capacity principle, conscious thought is constrained by its low capacity of consciousness. Unconscious thought does not have this constraint because the unconscious has a much higher capacity. It follows that conscious thought by necessity often takes into account only a subset of the information it should take into

\footnotetext{
${ }^{2}$ While the EV provides a clearer standard for the "best" option it does not take into account individual differences in (myopic) risk aversion (i.e., persons' willingness to pay for avoiding risks; Holt and Laury, 2002). We address this possible objection to our findings in the third experiment.
}

account" (Dijksterhuis and Nordgren, 2006, p. 96). The authors also refer to work on bounded rationality (Simon, 1955) which assumes that persons use simple strategies for information integration. Our interpretation of the principle is that UT is in general assumed to have higher capacity for information integration than conscious thought independent of whether information is still shown or has to be retrieved from memory (cf. Bröder and Schiffer, 2003; Glöckner and Hodges, 2011). In sufficiently complex tasks, this should lead to greater performance in UT relative to any condition employing conscious thought. Studies are, however, limited to comparing UT with conscious deliberation without information (Dw/oI, i.e., where the relevant information is not available for further examination). One might suspect that this specific situation is a particular challenge for conscious thought which needs to have access to the information during deliberation or it risks errors in memory retrieval (cf. Glöckner and Betsch, 2008; see also Glöckner and Herbold, 2011). We suspect that a similar argument holds for the UTE in that its apparent dominance of conscious deliberation will disappear if all information is provided to those deliberating consciously. This would indicate that eventual differences are not the result of capacity or the processing powers of the unconscious per se but due to information being absent, or distorted, during deliberation. We test the capacity principle of UTT with the following alternative hypothesis:

H1 - (Capacity Hypothesis): Unconscious thought will lead to better performance than conscious thought without information but not when compared to conscious thought with information.

The weighting principle is also stated rather generally in UTT: "According to the weighting principle, the unconscious naturally weights the importance of various attributes. Conscious thought often leads to suboptimal weighting because it disturbs this natural process" (Dijksterhuis and Nordgren, 2006, pp. 95-96). We interpret this to mean that UT supposedly leads to more accurate weighting of the choice options. Given this UTT would predict that UT will lead to better estimations of each of the choice options when compared to any condition involving conscious thought. Another alternative that is equally consistent with the reported findings above is that persons might just be better at detecting the best option during, or shortly after, information uptake and store a fuzzy representation of it until later retrieval (Reyna and Brainerd, 1995) unless such memories are degraded, or their retrieval made difficult, due to conscious thought without the information being available (Wilson and Schooler, 1991). If this were true, UT would fare reasonably well when selecting the best option but perform poorly if all options required evaluation. Specifically, one would expect that deliberation with information (DI) leads to a more accurate and differentiated evaluation of the options as compared to UT and conscious thought without information. These differential predictions motivate our second hypothesis. We test against the weighting principle of UTT with the following hypothesis:

H2 - (Differentiation Hypothesis) Conscious thought with information leads to more accurate and differentiated evaluation of the options as compared to unconscious thought.

Experiment 1 was conducted to investigate whether the standard UTE is found for gambling decisions comparing an UT condition and a Dw/oI condition. A new DI condition was added 
to the standard UT paradigm to more directly test the capacity principle of UTT. Participants were also asked to evaluate the EV of each of the gambles to test the appropriate weighting principle.

\section{EXPERIMENT 1 \\ METHOD}

\section{Participants}

Sixty-three participants (55\% female; age-range 16-38) from Bonn, Germany took part in Experiment 1. The experiment was run in combination with an unrelated study and participants were given on average $16 €$ (approximately US\$22.40) for their participation.

\section{Procedure and materials}

Participants started by reading instructions for the study which explained that their task would be to evaluate the EV of lotteries and to choose one lottery to play. They were told that in addition to a $3 €$ show-up payment they would receive up to $1 €$ per lottery if they correctly indicated the EV of the lottery and that this amount would decrease proportionally to the distance of their estimation. They were additionally informed that the lottery they selected would be incentivized and played out. Their understanding of what an EV is was tested by having them calculate the EV of a lottery with four outcomes similar to the ones used in the actual experiment. Only after providing a correct answer were they allowed to begin the computer-based experiment.

In the experiment participants selected between, and evaluated, 12 lotteries with 4 outcomes/attributes each (see Table 1). These gambles were randomly generated with the constraint that the payoffs be between 0 and $25 €$. We then randomly selected four gambles with EV's around $5 €, 10.00 €$, and $15.00 €$ (range: $\pm 1 €)$, to serve as our low, medium, and high EV gambles. Each of them had a specific name (i.e., Wynn, Venetian, Excalibur, Luxor, Rio, Flamingo, Mandalay, Sahara, Mirage, Bellagio, Platinum, and Ballys) and the assignment of names to lotteries was counterbalanced between participants. The experiment started with a presentation of each of the lotteries' outcomes and its probability (e.g., Wynn; $17.11 €, 35 \%$ ) one at a time for $5 \mathrm{~s}$ in random order

Table 1 | Gambles used in all three experiments ranked from highest to lowest EV. Each gamble consists of four probabilistic outcomes (attributes of the gambles).

\begin{tabular}{lrrrrr}
\hline Rank & Outcome 1 & Outcome 2 & Outcome 3 & Outcome 4 & EV \\
\hline 1 & $17.11 €, 0.35$ & $15.10 €, 0.49$ & $8.60 €, 0.12$ & $17.10 €, 0.04$ & $15.10 €$ \\
2 & $16.15 €, 0.32$ & $18.45 €, 0.31$ & $14.70 €, 0.24$ & $2.60 €, 0.13$ & $14.75 €$ \\
3 & $1.26 €, 0.34$ & $22.30 €, 0.28$ & $1.05 €, 0.04$ & $22.45 €, 0.34$ & $14.35 €$ \\
4 & $18.62 €, 0.37$ & $18.60 €, 0.06$ & $7.90 €, 0.21$ & $12.75 €, 0.36$ & $14.25 €$ \\
5 & $7.06 €, 0.22$ & $8.95 €, 0.60$ & $14.05 €, 0.03$ & $23.40 €, 0.15$ & $10.85 €$ \\
6 & $6.14 €, 0.37$ & $9.50 €, 0.27$ & $15.55 €, 0.02$ & $15.75 €, 0.34$ & $10.50 €$ \\
7 & $19.27 €, 0.19$ & $10.55 €, 0.49$ & $5.10 €, 0.03$ & $1.45 €, 0.29$ & $9.40 €$ \\
8 & $6.10 €, 0.13$ & $8.70 €, 0.31$ & $3.90 €, 0.31$ & $18.00 €, 0.25$ & $9.20 €$ \\
9 & $1.63 €, 0.09$ & $3.55 €, 0.15$ & $1.60 €, 0.36$ & $10.85 €, 0.40$ & $5.60 €$ \\
10 & $7.85 €, 0.14$ & $8.15 €, 0.23$ & $4.00 €, 0.40$ & $4.25 €, 0.23$ & $5.55 €$ \\
11 & $6.04 €, 0.31$ & $5.90 €, 0.12$ & $3.20 €, 0.50$ & $15.35 €, 0.07$ & $5.25 €$ \\
12 & $4.56 €, 0.27$ & $16.50 €, 0.13$ & $8.05 €, 0.04$ & $2.05 €, 0.56$ & $4.85 €$
\end{tabular}

(both by alternative and attribute) at the center of the screen. To allow time for proper encoding of the information each attribute presentation was separated by a 2 -s blank screen presentation.

After all attributes were presented, participants were placed into one of three treatment conditions, UT $(N=21)$, Dw/oI $(N=20)$, and DI $(N=23)$. In the UT condition participants completed a visual working memory task lasting $5 \mathrm{~min}$ after presentation of all lottery information (see Luck and Vogel, 1997 for a description of the task). In the Dw/oI condition participants were shown a blank screen with only the names of the 12 lotteries and asked to spend 5 min thinking about them. In the DI condition participants were given the same instructions but were presented with the outcomes and probabilities of each of the lotteries directly under their respective names for $5 \mathrm{~min}$. Participants then indicated the EV of each lottery, in random order, on a scale ranging from 0 to $25 €$. The final task was to select the lottery they wished to play.

\section{RESULTS}

To test the capacity hypothesis and to investigate whether UTE exists for gambles we tested whether choices were mainly made for one of the four high EV gambles and whether the highest EV gamble was chosen. We found that participants were generally able to select one of the best gambles in $67 \%$ of the cases which is significantly higher than chance $(p<0.01)$. In an overall test there was no difference between the conditions for selection of one of the four high EV gambles, Fishers exact $\chi^{2}(2,63)=1.31, p=0.52$. Looking only at the selection of the very best (i.e., the highest EV) gamble, a logistic regression showed that the UT condition outperformed the Dw/oI condition, odds $=0.18, z=-1.97, p<0.05$, which replicates the common UTE. In line with our first hypothesis, there was however no significant difference between the DI condition and the UT condition, $o d d s=0.57, z=-0.85, p=0.39$, counter to what UTT would predict (see Figure 1). It has, however,

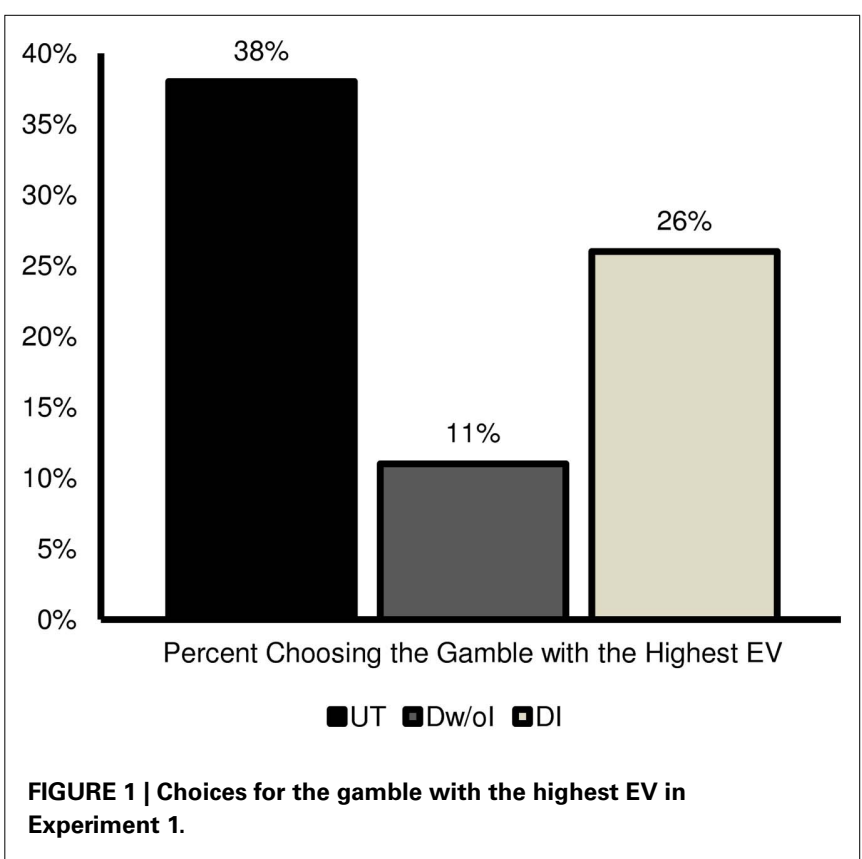


to be acknowledged that our analysis had only medium power ${ }^{3}$ and therefore the finding in favor of the null has to be interpreted cautiously.

To test the appropriate weighting principle of UTT we analyzed the difference in EV estimation using the absolute difference in estimation from the actual EV. Low numbers indicate more accurate estimations. A multi-level random coefficient model (Nezlek et al., 2006) with repeated judgments at level 1 and participants with random intercepts at level 2 revealed that the UT condition $(M=5.56, \mathrm{SE}=0.55$; high scores indicate bad performance) did not make significantly poorer estimations than the Dw/oI condition $(M=4.55, \mathrm{SE}=0.40), b=-1.01$, $z(63)=-1.66, p=0.09$, but did make significantly poorer estimations than the DI $(M=3.54, \mathrm{SE}=0.26)$ condition, $b=-2.03$, $z(63)=-3.44, p<0.01$ (see Figure 2). ${ }^{4,5}$

To additionally test whether the weighting principle might at least hold considering ordinal ranks, we calculated Spearman's rank order correlations for each subject between persons EV estimations and the true EV. We compared individual level correlations using $t$-tests on Fisher $Z$-transformed scores and found that persons in the UT condition $(\rho=0.18)$ did significantly worse in rank ordering the gambles than did participants in the DI condition $(\rho=0.38), t(42)=2.17, p=0.03$. We did not find the Dw/oI

\footnotetext{
${ }^{3}$ We used G* Power (Faul et al., 2007) to determine the power of our analysis. Assuming a medium effect size as reported for the UTE in Acker (2008) and Strick et al. (unpublished) we find that our analysis had a power of $1-$ beta $=0.51$.

${ }^{4}$ These results also hold if analyzing estimations of the gamble with the highest EVs only.

${ }^{5}$ The greatest estimation errors (i.e., average absolute EV-deviation) were observed in the four high EV gambles $(M=5.63$; $\mathrm{SE}=0.37)$, followed by the four low EV gambles $(M=4.20$; $\mathrm{SE}=0.40)$, and the smallest deviations occurring in the four medium EV gambles $(M=3.73$; $\mathrm{SE}=0.30)$.
}

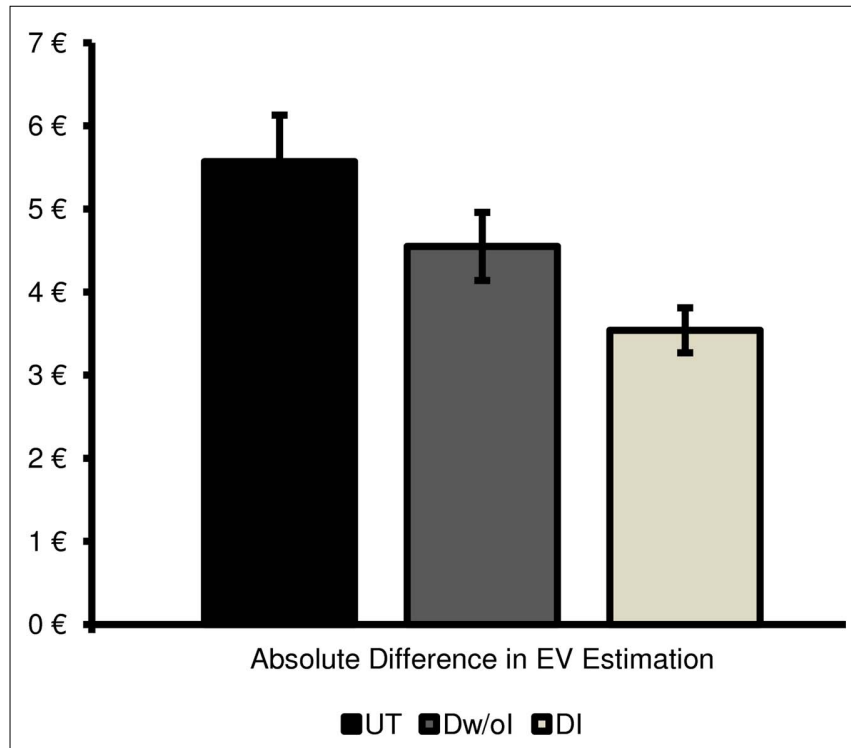

FIGURE 2 | Absolute deviations of EV estimations in Experiment 1. Higher scores indicate larger deviations and therefore less differentiated knowledge concerning the option. Error bars indicate SEs corrected for clusters at the participant level. condition $(\rho=0.19)$ to differ significantly from the UT condition, $p=0.86$. Hence, using two different methods to look at the ability to estimate and rank the EVs we find the data are in line with our second hypothesis and fail to support the weighting principle as put forth by UTT.

\section{DISCUSSION}

We find that the UT condition does better than the Dw/oI condition but not significantly better than the DI condition in selecting the gamble with the highest EV. Accordingly, in our task thinking unconsciously is only superior to thinking consciously when participants were not provided with information during the thinking phase. This fails to support the general capacity principle put forward by UTT but supports our alternative hypothesis. It appears that there is sufficient capacity for conscious deliberation to perform as well as its unconscious counterpart opposite to the core propositions put forth by UTT. As a caveat to this finding in favor of the null hypothesis it has, however, to be acknowledged that our analysis had only medium power (but see also further results in support of this hypothesis below). Interestingly, we could demonstrate the UTE with numerical gambles, which was doubted by Dijksterhuis et al. (2010). Our data show that this response was premature as we find some evidence for the "classic" UTE with gambles (i.e., comparing the conditions Dw/oI and UT).

As predicted by our differentiation hypothesis, despite doing well in gamble selection the UT condition was outperformed by the DI condition in estimating the EVs of each gamble and in rank ordering the gambles by EV. It seems as though the underlying mechanisms for these two tasks are different and favor unconscious processing only when the task structure and particularly the analyzed dependent variable is similar to what is commonly used in UT experiments: selecting the best choice without more detailed and differentiated judgments of all options being required.

\section{EXPERIMENT 2}

Experiment 2 aimed to replicate the results of Experiment 1 with an improved design that used conditions for which the UTE should be more likely to be found. Particularly, following Acker (2008) we used a word search task as the distracter task in the UT condition, shorter presentation intervals, and we reduced the time for un/conscious thought.

\section{METHOD \\ Participants}

Eighty participants (53\% female; age-range 17-66) from Bonn, Germany, took part in Experiment 2. The experiment was run in combination with an unrelated study and participants received on average $16 €$ for their participation.

\section{Procedure and materials}

Experiment 2 used the same lotteries and outcomes as Experiment 1 and followed the same basic pattern except for a few changes. Each outcome was presented for $2 \mathrm{~s}$ and the spacing between outcome presentations was shortened to $500 \mathrm{~ms}$. The distraction task for the UT $(N=27)$ condition was replaced with a word search task and each treatment condition, DI $(N=26)$ and Dw/oI $(N=27)$, was shortened to $4 \mathrm{~min}$. 


\section{RESULTS}

As in Experiment 1, no difference was found between the UT, Dw/oI, and DI conditions for selection of one of the four high EV gambles, Fishers exact $\chi^{2}(2,80)=2.33, p=0.34$. Looking only at the selection of the best gamble we find a significant difference between the UT, Dw/oI and, DI conditions, Fishers exact $\chi^{2}(2$, $80)=7.65, p=0.02$. A logistic regression revealed that the UT condition performed significantly better than the Dw/oI condition, odds $=0.16, z=-2.18, p=0.03$, but not significantly better than the DI condition, odds $=1.25, z=0.39, p=0.69$ (see Figure 3), thus replicating the standard UTE but also providing evidence against the generality of the capacity principle of UTT.

We analyzed the difference in EV estimation using the absolute difference in estimations from the EV as in Experiment 1. A multilevel random coefficient model with repeated judgments at level 1 and participants with random intercepts at level 2 revealed that the UT condition $(M=5.36, \mathrm{SE}=0.28)$ tended to perform worse than the DI condition $(M=4.64, \mathrm{SE}=0.41)$ but this difference did not reach conventional significance levels, $b=-0.71$, $z(79)=-1.47, p=0.07$, one-sided 6 . Nor, did the UT and Dw/oI $(M=5.56, \mathrm{SE}=0.31)$ conditions differ significantly, $p=0.67$ (see Figure 4).

As in Experiment 1 we performed Spearman rank order correlations between the subjective EV estimation and the true EV. We again found a tendency that DI shows the highest correlation but the differences were rather small and did not reach conventional significance levels (UT: $\rho=0.15$, DI: $\rho=0.18$, Dw/oI: $\rho=0.08$; all t's $<1.10$, all $p$ 's $>0.15$ ).

${ }^{6}$ Here and in the following analyses we used one-sided tests in cases in which we had derived a priori hypotheses based on findings from other studies reported in this paper that had been conducted before. Using one-sided tests is advisable under such conditions to avoid artificially increasing the beta error and decreasing the power of the analysis. All these instances are clearly marked in the text.

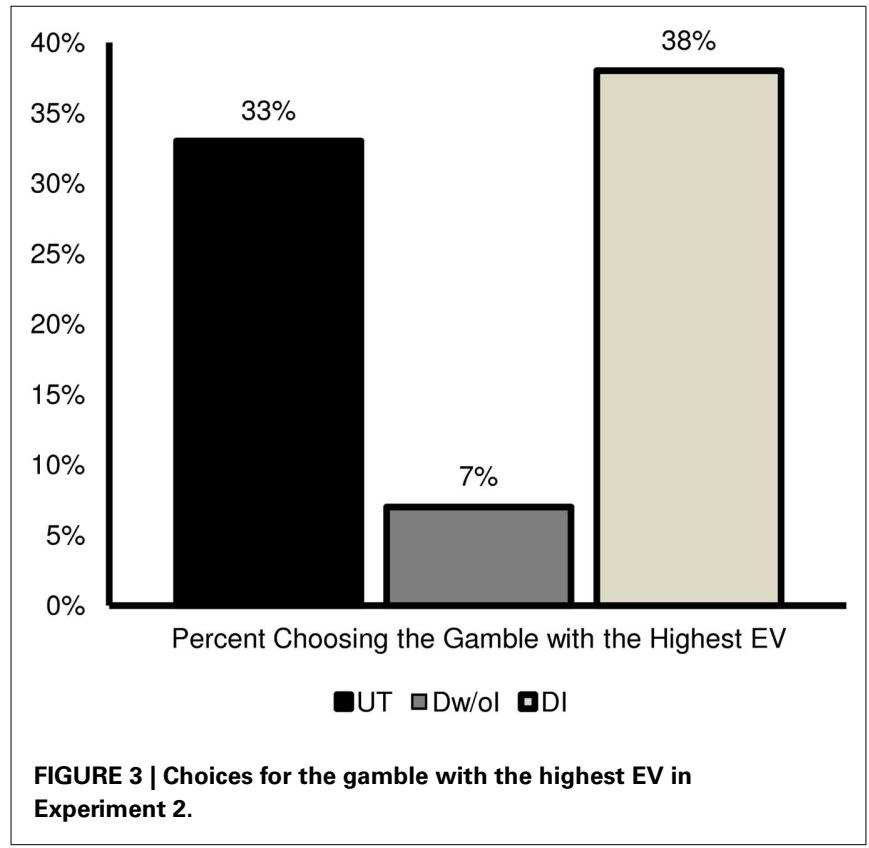

\section{DISCUSSION}

Experiment 2 replicates the results of Experiment 1 with UT performing as well as DI in making a good, or the best choice. The UT condition again outperforms the Dw/oI condition in choosing the best option. This provides a replication of the UTE found in Experiment 1 but at the same time speaks against the capacity assumption of UTT. Additionally, we again find that conscious deliberation with full information tends to produce closer estimations to the EV. It should, however, be noted that the effect was not significant at conventional levels for the interval scaled measure and even smaller for the rank-correlations (with arguably lower power). This fails to support the appropriate weighting assumption laid out in UTT but again tends to support our alternative differentiation hypothesis. However, it is worth noting that the slight changes made to the experimental paradigm seem to have resulted in a smaller difference between the UT, Dw/oI, and DI conditions in EV estimations.

\section{EXPERIMENT 3}

Although the results from Experiments 1 and 2 fail to support the capacity principle and the appropriate weighting principle put forth by UTT, it might still be asked whether the results also hold if people are not rewarded by playing the lottery they choose but instead if they are rewarded directly for choosing the lottery with the highest EV. This is important because as the criterion for best choices used in the previous experiments could still have suffered from differences in persons' risk preferences. This is the case because risk aversion can lead to preferring options with lower EV but also lower risk over options with higher EV but higher risk (Holt and Laury, 2002). Experiment 3 was designed to address this potential problem. We incentivized participants for selecting the gamble with the highest EV which fully removes any influences

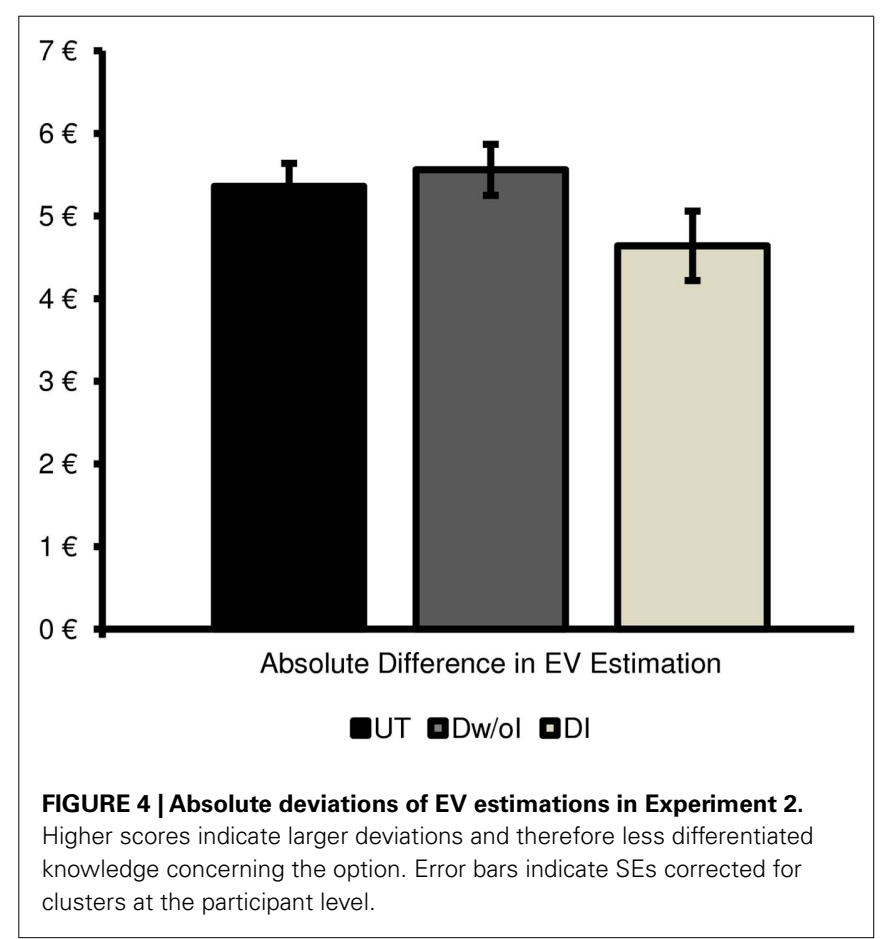


of risk preferences when selecting a gamble that might still have influenced results in Experiments 1 and 2. We also add a fourth immediate decision condition (I) to test whether the unconscious aspect of the UTE indeed has an influence on choice, or whether it can be equated to making a choice without deliberation.

\section{METHOD}

\section{Participants}

Seventy-four (61\% female; age-range 17-58) participants from Bonn, Germany, took part in Experiment 3. The experiment was run in combination with an unrelated study and participants earned on average $12 €$.

\section{Procedure and materials}

Experiment 3 used the same materials, procedure, and conditions as Experiment 2 with the slight modification that participants were told they would not play out the gamble they picked but instead earn $5 €$ if they selected the gamble with the highest EV. Additionally, the I condition was added in which participants indicated the gamble with the highest EV and made estimations of the gambles immediately following exposure to the gambles and their attributes.

\section{RESULTS}

No difference existed between the UT, Dw/oI, I, and DI conditions for selection of one of the four high EV gambles, Fishers exact $\chi^{2}(2,80)=2.29, p=0.51$. Looking only at the selection of the highest EV (i.e., the best) gamble we find in an overall test a significant difference between the UT, Dw/oI, I, and DI conditions, Fishers exact $\chi^{2}(3,74)=10.91, p=0.02$. A logistic regression revealed that the DI condition performed significantly better than the UT and I conditions (odds $=0.19, z=-2.25, p=0.03$ and odds $=0.09, z=-2.72, p<0.01$ respectively) and marginally better than the Dw/oI condition, odds $=0.28, z=-1.81, p=0.07$ (see Figure 5). Hence, with a clear incentive to choose the highest EV gamble the DI condition even outperformed the UT condition providing the strongest support for our capacity hypothesis $\mathrm{H} 1$ and against the capacity principle of UTT. No difference was found between the UT and I conditions, which fails to support any purported advantage of unconscious processing, odds $=0.47$, $z=-0.81, p=0.42$.

We again analyzed the difference in EV estimation using the absolute difference in estimations from the EV. A multi-level random coefficient model with repeated judgments at level 1 and participants with random intercepts at level 2 revealed that the UT condition $(M=4.96, \mathrm{SE}=0.26)$ fared significantly worse than the DI condition $(M=4.25, \mathrm{SE}=0.28), b=-0.71, z(73)=-1.77$, $p=0.04$, one-sided. The UT, Dw/oI $(M=5.09, \mathrm{SE}=0.34)$, and I $(M=4.89, \mathrm{SE}=0.22)$, conditions did not differ significantly from each other, all $p$ 's $>0.7$. The DI condition also performed better than the Dw/oI condition, $b=0.84, z(73)=2.10, p=0.02$, one-sided, and tended to be better than the I condition but the difference did not reach conventional significance levels, $b=0.64$, $z(73)=1.58, p=0.12$ (see Figure 6)

We again calculated Spearman rank order correlations between the participant subjective EV for the gambles and the true EV and found no differences in accuracy between the UT $(\rho=0.15)$, I
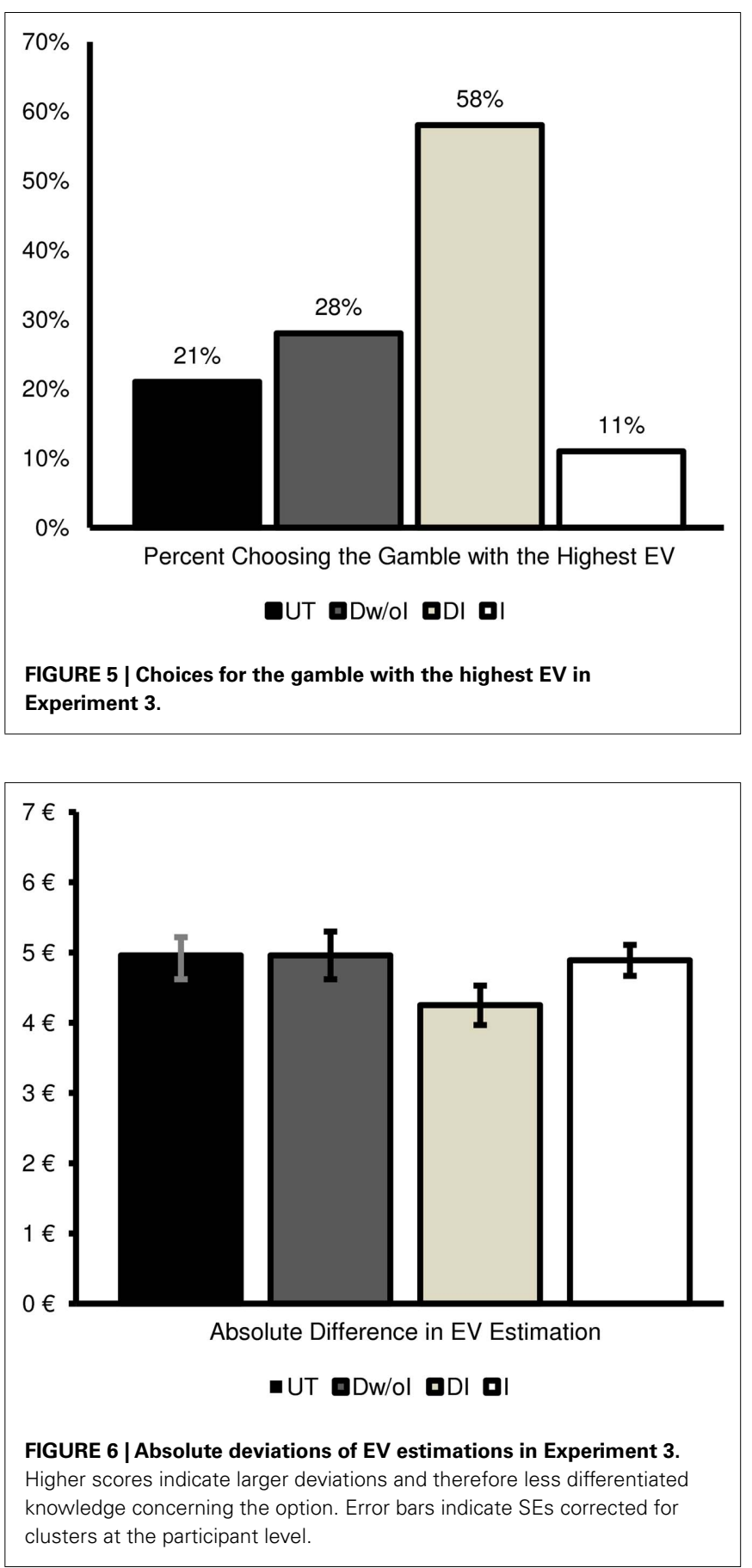

( $\rho=0.23)$, and Dw/oI $(\rho=0.13)$ conditions, $p$ 's $>0.3$. The DI condition $(\rho=0.28)$ tended toward better performance in ranking the EVs than the UT condition but this difference did not approach conventional significance levels, $t(36)=-1.34, p=0.10$, one sided.

\section{DISCUSSION}

Experiment 3 replicates the results of the first two studies. When it came to making a good choice, all four conditions did equally well. However, we do not find that the UT condition does better than any of the other conditions when selecting the best gamble and in 
fact did significantly worse when compared to the DI condition. This provides a third study arguing against the generality of the capacity principle put forth by UTT. The replication of the finding from Experiment 1 that the UT condition does worse than the DI condition in EV estimations and tends to be worse in rank ordering the gambles based on EV provides further evidence that the appropriate weighting principle put forth by UTT is questionable. We do not find a difference between the UT and I conditions in EV estimation or choosing the gamble with the highest EV which is in line with some previous findings indicating that there is not always an advantage to unconscious processing over making an immediate decision (Payne et al., 2008).

It should be noted that although rewarding for selection of the gamble with the highest EV removes influences of risk aversion, it might also induce participants to use a more deliberative form of information integration than they would naturally do. It has been shown that the combination of UT and the induction of a more intuitive decision mode improve performance in judgments (Usher et al., 2011). We cannot rule out that these specifics of our experimental setting might have degraded, or hampered, the performance of the unconscious. Given that, it should be kept in mind that the results of Experiment 3 might not generalize to settings in which more intuitive modes of thought are induced.

\section{GENERAL DISCUSSION}

The three experiments presented in this paper serve as an extension of previous findings on the UTE and provide tests of the generality of two key assumptions behind UTT. We find the classic UTE between the unconscious and Dw/oI conditions when it came to making the best choice in Experiments 1 and 2. UTE was present even when the choices and attributes involved purely numerical information, which speaks against limiting the UTE to decision tasks not involving numbers (Dijksterhuis et al., 2010). Our experiments suggest that - when accepting EV as comparison standard - the unconscious can deal with numerical information just as well as deliberative thought with full information being present.

The UT condition outperforms the Dw/oI condition which is in line with literature showing that conscious thought in the absence of information often leads to memory retrieval errors and overwrites (Schooler and Engstler-Schooler, 1990). As we have explained above, the comparison between unconscious thought and Dw/oI is, however, not the best test of UTT and its assumption that there is an inherent capacity advantage of unconscious over conscious deliberation. A more pragmatic comparison, and one which provides a true test of the capacity principle of UTT, is between the unconscious thought and the deliberation with full information condition. In all three experiments we find that unconscious thought is not superior, and in some cases even inferior, to deliberation with full information in making a good, or the best choice. In fact, if we jointly consider data for Experiments 1 and 2 we find that we achieve sufficient power ${ }^{7}$ to detect such an

\footnotetext{
${ }^{7}$ Again, using $G^{*}$ Power (Faul et al., 2007) to determine the power of our analysis and assuming a medium effect size we find that our analysis had a power of 1 - beta $=0.84$, although we fail to find the UT condition outperforming the DI condition as predicted by UTT, Fishers exact $\chi^{2}(1,97)=0.08, p=0.83$.
}

effect if present but fail to do so. These results are directly in line with our alternative capacity hypothesis stating that unconscious thought outperforms Dw/oI, but is not better than deliberation with full information.

With regard to EV estimations and rank ordering of the EVs the DI condition made smaller errors than the unconscious thought condition suggesting that while the unconscious may be able to remember and select a good, or the best choice, the information held and manipulated by the unconscious is highly limited in its detail. It might rely heavily on gist-like representations (Rivers et al., 2008) and might be hampered by analytic thought processes (cf. Usher et al., 2011). This finding provides strong support for our second hypothesis suggesting that the unconscious does not generally lend itself to appropriate weighting of information in evaluating all options. When evaluating the generality of our findings, it has to be kept in mind, however, that we used in all reported studies choices between gambles. These consist of abstract outcomes and probabilities and can in principle be solved in a rule based manner. Both factors are likely to induce a more deliberate mode of thought and might give some advantage to the conscious thought condition. It is due to further research to investigate whether the unconscious outperforms even deliberation with full information concerning choice and/or accuracy of evaluations in other domains involving less abstract and harder to grasp information.

\section{CONCLUSION}

The comparison between unconscious thought and Dw/oI shows that unconscious thought leads to better performance in more cases than conscious deliberation without the information being available. This is supported both from the studies presented here but also from other studies (e.g., Dijksterhuis et al., 2006; de Vries et al., 2010; Strick et al., 2010).

However, while such findings are interesting, we feel that such a comparison does not mirror many typical real-world situations individuals are placed in daily. In many situations such as important purchasing decisions individuals have the ability to reassess and study information about their options before making a decision. The comparison between the DI and unconscious thought conditions provides critical tests of such situations and at the same time allows testing the generality of basic principles postulated by UTT. In order for unconscious thought to be touted as the best strategy for making complex decisions with meaningful outcomes it must consistently outperform the DI condition. Although it has to be acknowledged that UT does surprisingly well, we do not find this superiority over DI to be the case in any of the experiments reported here. Given that we find a significant difference between the DI and UT with regards to selecting a good, or "best," option only in Experiment 3, but not in Experiments 1 and 2, we have to conclude that DI does not seem to be generally superior to UT; which is in itself an interesting finding given that in DI persons were given all chances to perform well. The difference in findings between Experiments 1 and 2 vs. 3 might be due to the fact that the two former studies could be understood as decisions of personal preference whereas detecting the highest EV gamble in the last study could be perceived as an inference task which might increase the probability of using deliberate 
calculations in the DI condition. More generally speaking, it is quite likely that in situations that involve numerical cues as the ones used in this study there may be no superiority, and in some cases that induce calculation as in Experiment 3, an inferiority of decisions stemming from unconscious thought compared to those coming from conscious thought with information available. However, in other situations (e.g., judgments of character, trustworthiness, or personality) in which cues are harder to capture in abstract terms but can be more easily grasped intuitively, or in cases where all pertinent information may not be available for review, the UT condition may in fact be found to outperform conscious DI.

According to Dijksterhuis (2004, p. 597), “[w]hen faced with complex decisions such as where to work or where to live, do not think too much consciously. Instead, after a little initial conscious information acquisition, avoid thinking about it consciously. Take

\section{REFERENCES}

Acker, F. (2008). New findings on unconscious versus conscious thought in decision making: additional empirical data and meta-analysis. Judgm. Decis. Mak. 3, 292-330.

Bos, M. W., Dijksterhuis, A., and van Baaren, R. B. (2008). On the goal dependency of unconscious thought. J. Exp. Soc. Psychol. 44, 1114-1120.

Bröder, A., and Schiffer, S. (2003). Take the best versus simultaneous feature matching: probalistic inferences form memory and effects of representation format. J. Exp. Psychol. Gen. 132, 277-293.

Calvillo, D. P., and Penaloza, A. (2009). Are complex decisions bettter left to the unconscious? Further failed replications of the deliberationwith-out attention effect. Judgm. Decis. Mak. 4, 509-517.

de Vries, M., Witteman, C. L. M., Holland, R. W., and Dijksterhuis, A. (2010). The unconscious thought effect in clinical decision making: an example in diagnosis. Med. Decis. Mak. 30, 578-581.

Dijksterhuis, A. (2004). Think different: the merits of unconscious thought in preference development, and decision making. J. Pers. Soc. Psychol. 87, 586-598.

Dijksterhuis, A., Bos, M. W., Nordgren, L. F., and van Baaren, R. B. (2006). On making the right choice: the deliberation without attention effect. Science 311, 1005-1007.

Dijksterhuis, A., Bos, M. W., van der Leij, A., and van Baaren, R. B. (2010). Predicting soccer matches after unconscious and conscious thought as a function

your time and let the unconscious deal with it." Although we do not want to question phenomena such as memory consolidation by sleeping (e.g., Gais and Born, 2004), we find such suggestions to do little initial conscious information acquisition to be over-generalized and possibly damaging when the decisions mentioned have far reaching consequences. Until the UTE is further explored and its underlying processes (see, e.g., Usher et al., 2011) and boundary conditions clearly understood we suggest that when faced with decisions that involve abstract information individuals will perform their best when they gather all the information they can about a decision and take the time to study that information and consciously compare the outcomes before making a choice based on careful deliberation and emerging intuition (see, e.g., Janis and Mann, 1977). Our results indicate that in such decisions with abstract information doing so does not hurt and sometimes even increases performance in judgment and choice.

of expertise. Psychol. Sci. 20, González-Vallejo, C., Lassiter, G. D., 1381-1387.

Dijksterhuis, A., and Nordgren, L. F. (2006). A theory of unconscious thought. Perspect. Psychol. Sci. 1, 95-109.

Dougherty, M. R. P., Gettys, C. F., and Ogden, E. E. (1999). MINERVADM: a memory processes model for judgments of likelihood. Psychol. Rev. 106, 180-209.

Evans, J. S. B. T. (2008). Dual-processing accounts of reasoning, judgment, and social cognition. Annu. Rev. Psychol. 59, 255-278.

Faul, F., Erdfelder, E., Lang, A.-G., and Buchner, A. (2007). G*Power 3: a flexible statistical power analysis program for the social, behavioral, and biomedical sciences. Behav. Res. Methods 39, 175-191.

Gais, S., and Born, J. (2004). Declarative memory consolidation: mechanisms acting during human sleep. Learn. Mem. 11, 679-685.

Glöckner, A., and Betsch, T. (2008). Multiple-reason decision making based on automatic processing. J. Exp. Psychol. Learn. Mem. Cogn. 34, 1055-1075.

Glöckner, A., and Herbold, A. K. (2011). An eye-tracking study on information processing in risky decisions: evidence for compensatory strategies based on automatic processes. J. Behav. Decis. Mak. 24, 71-98.

Glöckner, A., and Hodges, S. D. (2011). Parallel constraint satisfaction in memory-based decisions. Exp. Psychol. 58, 180-195.

Glöckner, A., and Witteman, C. L. M. (2010). Beyond dual-process models: a categorization of processes underlying intuitive judgment and decision making. Thinking Reason. 16, 1-25.
Bellezza, F. S., and Lindberg, M.

J. (2008). "Save angels perhaps": a critical examination of unconscious thought theory and the deliberationwithout-attention effect. Rev. Gen. Psychol. 12, 282-296.

González-Vallejo, C., and Phillips, N. (2010). Predicting soccer matches: a reassessment of the benefit of unconscious thinking. Judgm. Decis. Mak. 5, 200-206.

Ham, J., van den Bos, K., and van Doorn, E. (2009). Lady justice thinks unconsciously: unconscious thought can lead to more accurate justice judgments. Soc. Cogn. 27, 510-522.

Holt, C. A., and Laury, S. K. (2002). Risk aversion and incentive effects. Am. Econ. Rev. 92, 1644-1655.

Janis, I. L., and Mann, L. (1977). Decision Making: A Pschological Analysis of Conflict, Choice, and Commitment. New York: The Free Press. capacity of visual working memory for features and conjunctions. Nature 390, 279-281. E. (1981). An interactive activation model of context effects in letter perception: I. An account of basic findings. Psychol. Rev. 88, 375-407.

Miller, G. A. (1956). The magical number seven, plus or minus two: some limits on our capacity for processing information. Psychol. Rev. 63, 81-97.

Nezlek, J. B., Schröder-Abe, M., and Schütz, A. (2006). Multilevel analyses in psychological research: advantages and potential of multilevel random coefficient modeling. Psychol. Rundsch. 57, 213-223.

Payne, J. W., Samper, A., Bettman, J. R., and Luce, M. F. (2008). Boundary
Luck, S. J., and Vogel, E. K. (1997). The

McClelland, J. L., and Rumelhart, D. conditions on unconscious thought in complex decision making. Psychol. Sci. 19, 1118-1123.

Rey, A., Goldstein, R. M., and Perruchet, P. (2009). Does unconscious thought improve complex decision making? Psychol. Res. 73, 377-379.

Reyna, V. F., and Brainerd, C. J. (1995). Fuzzy-Trace theory: some foundational issues. Learn. Individ. Differ. 7, 145-162.

Rivers, S. E., Reyna, V. F., and Mills, B. (2008). Risk taking under the influence: a fuzzy-trace theory of emotion in adolescence. Dev. Rev. 28, 107-144.

Schooler, J. W., and Engstler-Schooler, T. Y. (1990). Verbal overshadowing of visual memories: some things are left better off unsaid. Cogn. Psychol. 22, 36-71.

Simon, H. A. (1955). A behavioural model of rational choice. Q. J. Econ. 69, 99-118.

Smith, P. K., Dijksterhuis, A., and Wigboldus, D. H. J. (2008). Powerful people make good decisions even when they consciously think. Psychol. Sci. 19, 1258-1259.

Strick, M., Dijksterhuis, A., and van Baaren, R. B. (2010). Unconcious thought effects take place offline, not on-line. Psychol. Sci. 21, 484-488.

Thomas, R. P., Dougherty, M. R., Sprenger, A. M., and Harbison, J. I. (2008). Diagnostic hypothesis generation and human judgment. Psychol. Rev. 115, 155-185.

Thorsteinson, T. J., and Withrow, S. (2009). Does unconscious thought outperform conscious thought in complex decisions? A further examination. Judgm. Decis. Mak. 4, 235-247. 
Tordesillas, R., and Chaiken, S. (1999). Thinking too much or too little? Pers. Soc. Psychol. Bull. 25, 625-631.

Tversky, A., and Kahneman, D. (1974). Judgment under uncertainty: heuristics and biases. Science 185, 1124-1131.

Usher, M., and McClelland, J. L. (2001). The time course of perceptual choice: the leaky, competing accumulator model. Psychol. Rev. 108, 550-592.

Usher, M., Russo, Z., Weyers, M., Brauner, R., and Zakay, D. (2011). The impact of mode of thought in complex decisions: intuitive decisions are better. Front. Psychol. 2:37. doi:10.3389/fpsyg.2011.00037
Waroquier, L., Marchiori, D., Klein, O., and Cleeremans, A. (2009). Methodological pitfalls of the unconscious thought paradigm. Judgm. Decis. Mak. 4, 601-610.

Waroquier, L., Marchiori, D., Klein, O., and Cleeremans, A. (2010). Is it better to think unconsciously or to trust your first impression? A reassessment of unconscious thought theory. Soc. Psychol. Pers. Sci. 1, 111-118.

Wilson, T. D., Lisle, D. J., Schooler, J. W., Hodges, S. D., Klaaren, K. J., and LaFleur, S. J. (1993). Introspecting about reasons can reduce postchoice satisfaction. Pers. Soc. Psychol. Bull. 19, 331-339.
Wilson, T. D., and Schooler, J. W. (1991). Thinking too much: introspection can reduce the quality of preferences and decisions. J. Pers. Soc. Psychol. 60, 181-192.

Conflict of Interest Statement: The authors declare that the research was conducted in the absence of any commercial or financial relationships that could be construed as a potential conflict of interest.

Received: 09 May 2011; accepted: 19 September 2011; published online: 10 October 2011.
Citation: Ashby NJS, Glöckner A and Dickert S (2011) Conscious and unconscious thought in risky choice: testing the capacity principle and the appropriate weighting principle of unconscious thought theory. Front. Psychology 2:261. doi: 10.3389/fpsyg.2011.00261

This article was submitted to Frontiers in Cognitive Science, a specialty of Frontiers in Psychology.

Copyright (C) 2011 Ashby, Glöckner and Dickert. This is an open-access article subject to a non-exclusive license between the authors and Frontiers Media $S A$, which permits use, distribution and reproduction in other forums, provided the original authors and source are credited and other Frontiers conditions are complied with. 